\title{
Consumo de
}

sustancias

psicoactivas y

riesgo suicida en

usuarios de centros

de rehabilitación de

adicciones

Amador, Salinas y Pérez 
Consumo de sustancias psicoactivas y riesgo suicida en usuarios de centros de rehabilitación de adicciones

Psychoactive substance use and suicidal risk in users of addiction rehabilitation

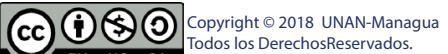

MSc. Dustin Ezequiel Amador Jiménez Master en Psicología Clínica y de la Salud, Licenciado en Psicología, profesor del Departamento de Psicología, Universidad Nacional Autónoma de Nicaragua (UNANManagua).

https://orcid.org/0000-0001-6894-4997

dustin_amador@live.com

Lic. Xochilt María Salinas Pérez.

Licenciada en Psicología, egresada del Departamento de Psicología de la UNANManagua.

https://orcid.org/0000-0003-1847-1217 xo0150508@gmail.com

Lic. Cristhian Josué Pérez López. Licenciado en Psicología, egresado del Departamento de Psicología de la UNAN-

Recibido: 20 de enero 2018

Aceptado: 10 de marzo 2018

\section{RESUMEN}

El estudio tiene como objetivo evaluar la relación entre el consumo de sustancias psicoactivas y riesgo suicida en un grupo de consumidores de Sustancias Psicoactivas en rehabilitación durante el primer semestre del año 2018. Método: En el estudio se utilizó un enfoque cuantitativo, el cual permitió describir y establecer la relación de las variables en estudio; se utilizó un diseño no experimental, de corte transversal, con un alcance descriptivocorrelacional. La muestra está conformada por 54 usuarios pertenecientes a los centros de rehabilitación CARA, CENICSOL, HODERA y Grupo A.A. Hermandad 25 de noviembre. Se aplicó la Escala de Riesgo Suicida de Plutchik (RS) y la Prueba de detección de consumo de alcohol, tabaco y sustancias (ASSIST). Se hizo uso de estadística descriptiva y estadística inferencial, la cual permitió realizar el contraste de hipótesis. Resultados: Los resultados indican que la principal droga impacto de los participantes es el alcohol. En cuanto al riesgo suicida de los usuarios, se comprobó que la mayoría se encuentran en riesgo leve, es decir que experimentan ideaciones suicidas. Finalmente, se comprobó que existe relación estadísticamente significativa entre el consumo de sedantes y cannabis con el riesgo suicida.

\section{PALABRAS CLAVE}

Riesgo suicida. Consumo de sustancias psicoactivas. Sedantes. Cannabis. 


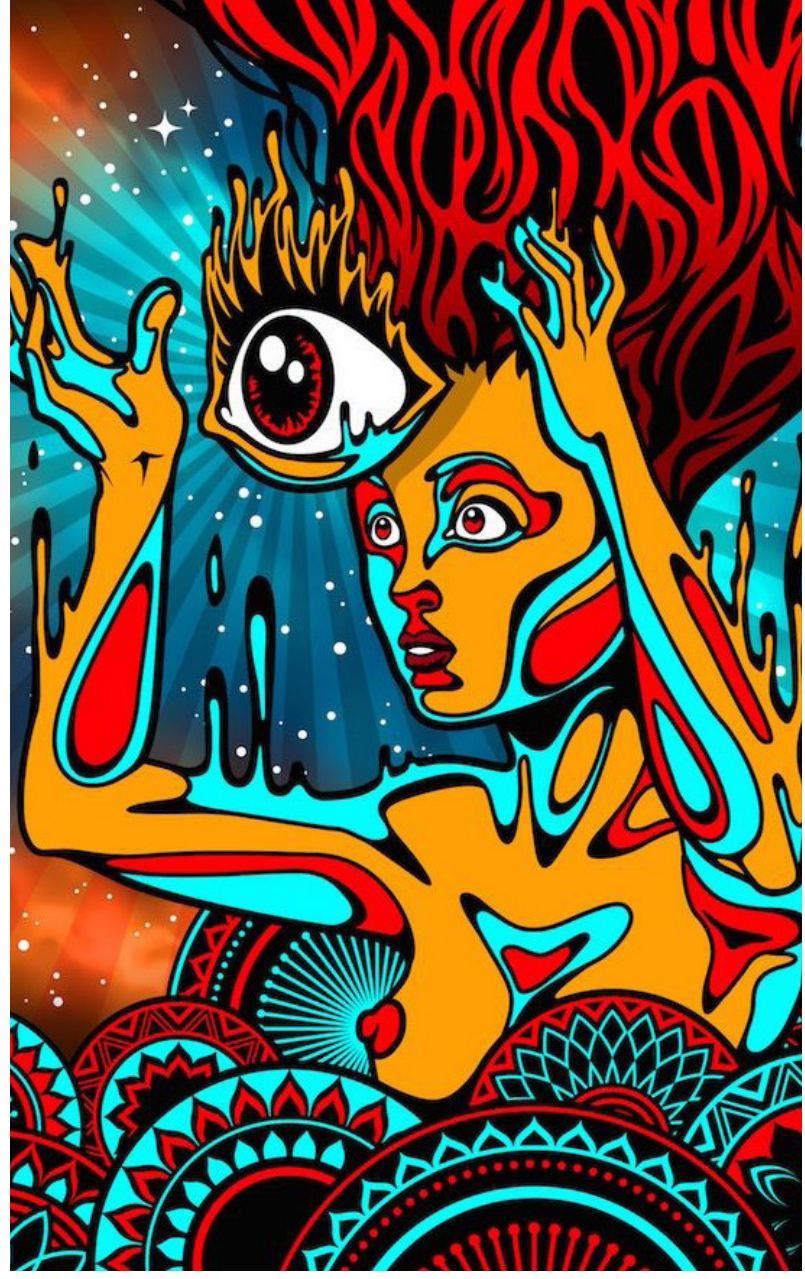

\section{ABSTRACT}

The study aims to evaluate the relationship of psychoactive substance use and suicidal risk in a group of users of addiction rehabilitation centers during the first half of 2018. Method: The study used a quantitative approach, which allowed the description and establishment of the relationship of the variables under study; a non-experimental, cross sectional design was used, with a descriptive-correlational scope. The participants were 54 users of the rehabilitation centers CARA, CENICSOL, HODERA and the A.A. group Hermandad 25 de noviembre. The Plutchik Suicide Risk Scale (RS) and the Alcohol, Tobacco and Substances Screening Test (ASSIST) were applied. Descriptive and inferential statistics were used, which allowed the hypothesis contrast to be realized. Results: Results indicate that the main impact drug of participants is alcohol. As for the suicidal risk, it was found that most are at mild risk; it means that they experience suicidal thought. Finally, it was found that there is a statistically significant relationship between sedative drugs and cannabis use with suicidal risk. 


\title{
Introducción
}

\begin{abstract}
$\mathrm{E}$ I presente estudio tiene como objetivo principal evaluar la relación entre el consumo de sustancias psicoactivas y el riesgo suicida en un grupo de consumidores en rehabilitación durante el primer semestre del año 2018, para ello se debe determinar el nivel de riesgo de consumo de diversas sustancias y el nivel de riesgo suicida de los participantes. El estudio fue realizado en el Centro Nicaragüense de Solidaridad (Cenicsol), el Centro de Ayuda y Rehabilitación del Alcohólico (CARA), El grupo de Alcohólicos Anónimos (AA) Hermandad 25 de noviembre de la ciudad de Managua y el Hogar HODERA de la ciudad de San Marcos, Carazo. Estos centros se encargan de la atención, rehabilitación y reinserción social de personas que manifiesten problemas de abuso de sustancias psicoactivas.
\end{abstract}

El estudio evalua el consumo de sustancias psicoactivas y el riesgo suicida. La OMS (2008) las define como sustancias naturales o químicas, que introducidas en un organismo vivo son capaces de actuar sobre el Sistema Nervioso Central y producir un cambio en la conducta de las personas. El consumo prolongado y abuso de diversas sustancias se ha asociado al deterioro clínicamente significativo de distintas áreas de la vida de las personas (APA, 2014). Entre las consecuencias negativas se encuentra el incremento del riesgo suicida, el cual es un fenómeno compuesto por la presencia de ideas, planes y conductas suicidas. Diversos estudios internacionales (Aristizábal, et al., 2012; Gonzales C. et al, 2015; Martínez, 2006) sugieren como hipótesis que el consumo de sustancias psicoactivas tiene relación con la conducta suicida, por lo que es necesario estudiar detenidamente si existe una correlación significativa entre estas variables en el contexto nicaragüense. Con esta información las instituciones encargadas de abordar este tipo de problemáticas podrán diseñar planes terapéuticos pertinentes basados en evidencia científica.

\section{Métodos}

Tipo de estudio: El estudio es una investigación aplicada, se utilizó un enfoque cuantitativo, el cual permitió describir y establecer la relación de las variables en estudio; se utilizó un diseño no experimental, de corte transversal, con un alcance descriptivo-correlacional. Participantes: La muestra está compuesta por 54 sujetos adultos de ambos sexos consumidores de sustancias psicoactivas en rehabilitación en los centros CENICSOL, CARA, HODERA y Grupo AA Hermandad 25 de noviembre. El muestreo es de tipo no probabilístico o dirigido, ya que la elección de los participantes no depende de la probabilidad sino de las características de la investigación. Instrumentos: Se aplicó la Escala de Riesgo Suicida de Plutchik (RS) y la Prueba de detección de consumo de alcohol, tabaco y sustancias (ASSIST). Procedimiento: Para el análisis de los datos obtenidos se empleó procedimientos estadísticos descriptivos como Distribución de frecuencia; para realizar la prueba de hipótesis y establecer el nivel de relación estadística entre las variables se empleó estadística inferencial, con las pruebas no paramétricas Chi cuadrada (X2) y la Prueba exacta de Fisher, en las que se asumió un Nivel de confianza del $95 \%(\infty=0.05)$. 


\section{Resultados}

Abuso de sustancias: De acuerdo a la APA (2014), los trastornos por consumo de sustancias psicoactivas implican una asociación de síntomas cognitivos, comportamentales y fisiológicos que muestran que la persona continúa consumiendo a pesar de los problemas significativos generados por la sustancia.

En base a lo anterior, la droga de impacto es la sustancia psicoactiva que produce mayores efectos negativos en la vida del consumidor en áreas diversas de su vida y es la que lo impulsa a buscar ayuda o tratamiento para su adicción. En la Figura 1 se puede observar los resultados con respecto a esta variable.

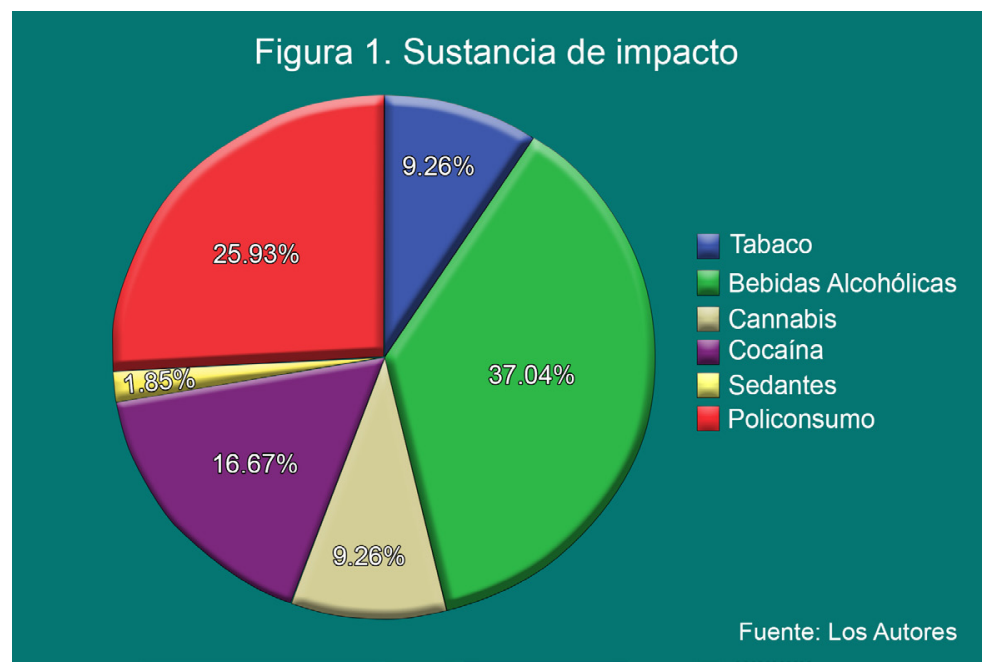

Al aplicar el ASSIST, se identificó que el 37.04\% de los sujetos de estudio consumen bebidas alcohólicas, la cual es la droga que genera mayor riesgo. Otros datos destacables dentro de estos resultados es que el $25.93 \%$ de los participantes son policonsumidores (abusan de más de una sustancia) y otro $16.67 \%$ son consumidores de cocaína (crack).

Nivel de Riesgo: El nivel de riesgo se refiere a los patrones de consumo desustancias que indica el peligro de sufrir consecuencias nocivas para el sujeto. En este caso, se presentan las sustancias con mayores niveles de riesgo.

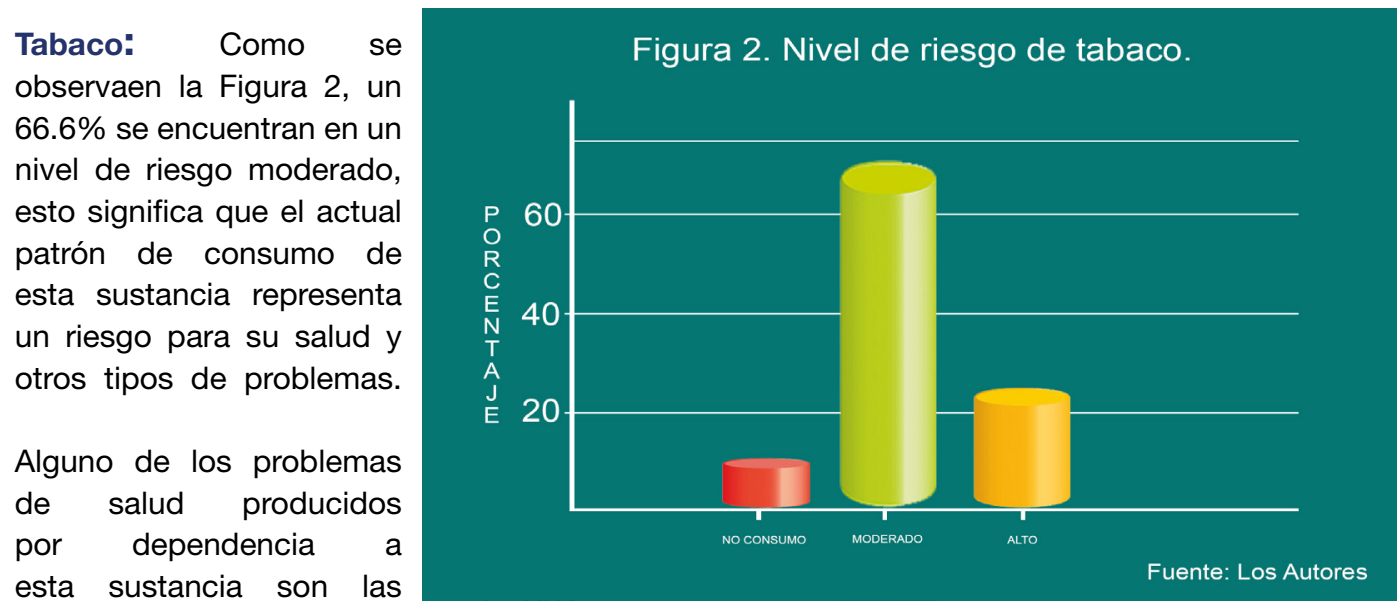

enfermedades respiratorias, asma, alergias, enfermedad cardiovascular y cánceres (OMS, 2011). 
Bebidas alcohólicas: Los resultados de a Figura 3 indican que el 59.26\% de los participantes se encuentran en nivel de riesgo alto, lo que significa una posible experimentación de problemas graves de salud, sociales, económicos e incluso legales. Además, el $35.19 \%$ de los participantes se encuentran en un nivel de riesgo moderado, lo que refiere que su actual patrón de consumo de sustancias representa un riesgo para su salud y otro tipo de problemas. Algunos de los problemas asociados son resacas, conductas agresivas, pancreatitis, hipertensión arterial, ansiedad y depresión, dificultades cognitivas, lesión cerebral, cánceres y suicidio.

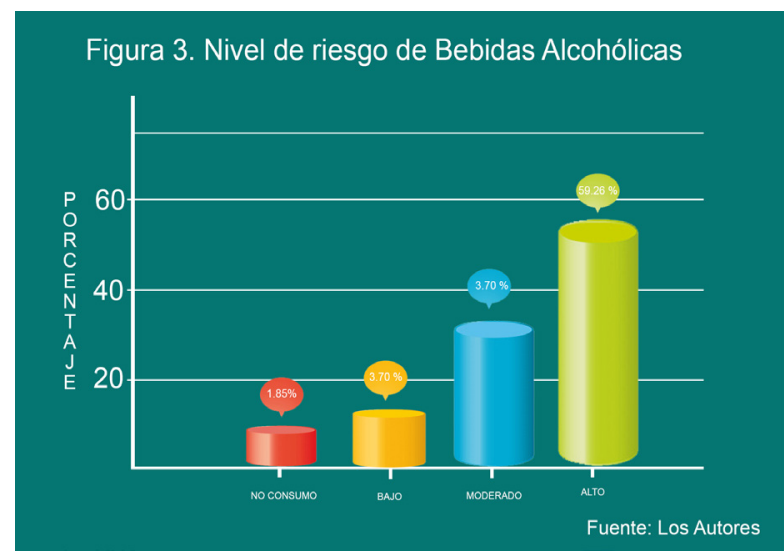

Cannabis: La Figura 4 presenta información sobre el nivel de riesgo del cannabis, se observa que el $35.1 \%$ de los participantes del estudio se encuentran en un nivel de riesgo moderado y solo un $25.93 \%$ de los participantes en riesgo alto, estos tipos de riesgo desencadena en la persona problemas graves de salud, sociales, económicos, legales y de pareja.

Algunos de los problemas que representa el consumo de esta sustancia psicoactiva son dificultades con la atención y motivación, ansiedad, paranoia, pánico, depresión, pérdida de memoria y en la capacidad de

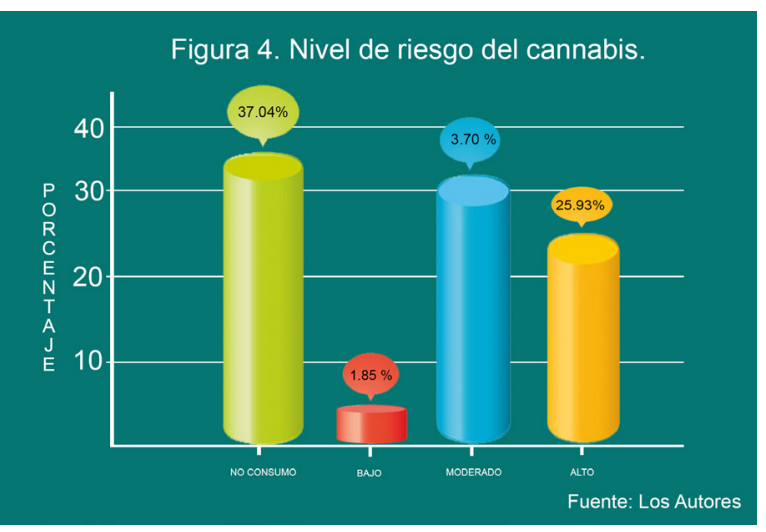
solución de problemas, enfermedad cardíaca, enfermedad respiratoria obstructiva crónica y cánceres (ibid).

Cocaína: En la Figura 5 se representan los datos obtenidos sobre el nivel de riesgo de cocaína,el $38.89 \%$ tienen un nivel de riesgo alto reflejando problemas graves de salud $y$ un $37.04 \%$ se encuentran en un nivel de riesgo moderado. De acuerdo con estos resultados, las personas consumidoras de esta sustancias; se encuentran vulnerable a experimentar dificultades para dormir, dolores de cabeza, pérdida de peso, sensaciones de adormecimiento, problemas económicos, pensamientos irracionales, ansiedad, depresión, manía, agresividad, paranoia, ansias de consumo,

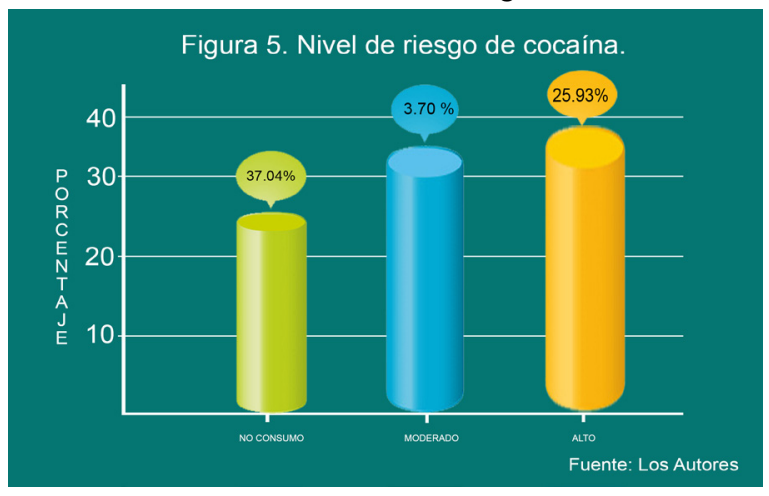
estrés, incluso psicosis o muerte súbita debido a problemas cardíacos tras el consumo repetido de altas dosis (ibid). 
Sedantes: La Figura 6 presenta información sobre el nivel de riesgo de sedantes, de acuerdo con los datos adquiridos, un $18.5 \%$ de los participantes tienen un nivel de riesgo moderado, lo que indica que su actual patrón de consumo de esta sustancia representa un riesgo para su salud y que necesitan asistir a un programa de intervención. Asimismo, pueden experimentar estados de confusión, mareo, sedación, dolor de cabeza, inestabilidad al andar, problemas de sueño, ansiedad y depresión, síntomas de abstinencia, tolerancia y dependencia después de un período breve de consumo.

Niveles de riesgo suicida: Se entiende como riesgo suicida a la probabilidad que tiene una persona de idear o intentar quitarse la vida (Rocamora, 2013). Este tipo de conducta se entiende como un proceso continuo que va desde las ideas en sus diferentes expresiones, pasando por las amenazas, los intentos y culminando en el suicidio propiamente dicho.

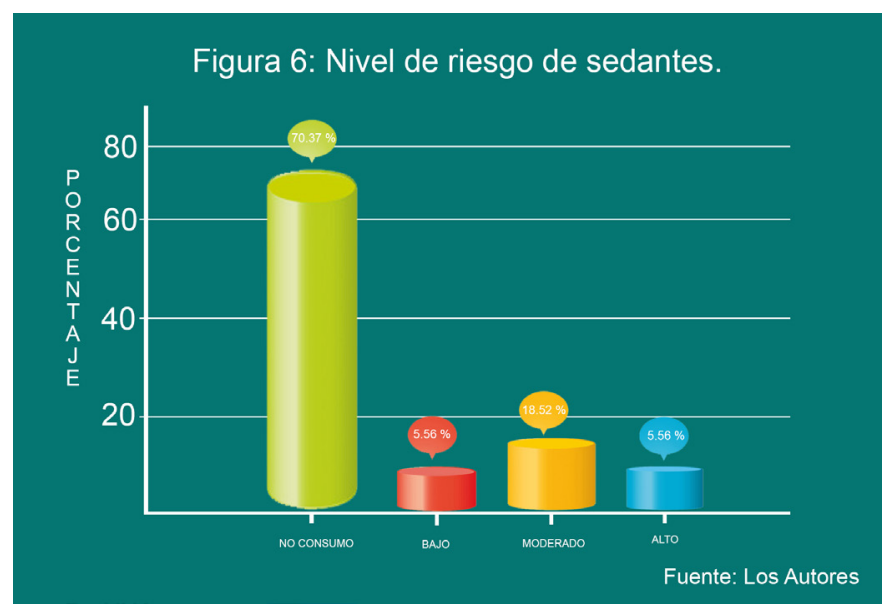

En la Figura 7 se puede observar los resultados obtenidos en referencia al riesgo suicida de los participantes, el $44.4 \%$ de los usuarios se encuentran en un nivel de riesgo bajo, lo que significa que no ha habido intentos suicidas, sin embargo, estas personas si han experimentado ideación suicida con frecuencia, intensidad y duración bajas, la persona que se encuentra en este nivel de riesgo suele tener pensamientos como "No puedo salir adelante", "desearía estar muerto", y a pesar de no haber una planificación, es necesario que la persona tenga ayuda profesional.

En esta Figura también se observa que el $40.7 \%$ de los participantes tiene un nivel de riesgo moderado, indicando que las ideas suicidas son más intensas, frecuentes y persistentes. Es posible que existan planes vagos, pero no hay intención explícita de cometer suicidio.

Los síntomas son moderados y aún existe buen autocontrol, no obstante, es

\section{Figura 7. Riesgo}

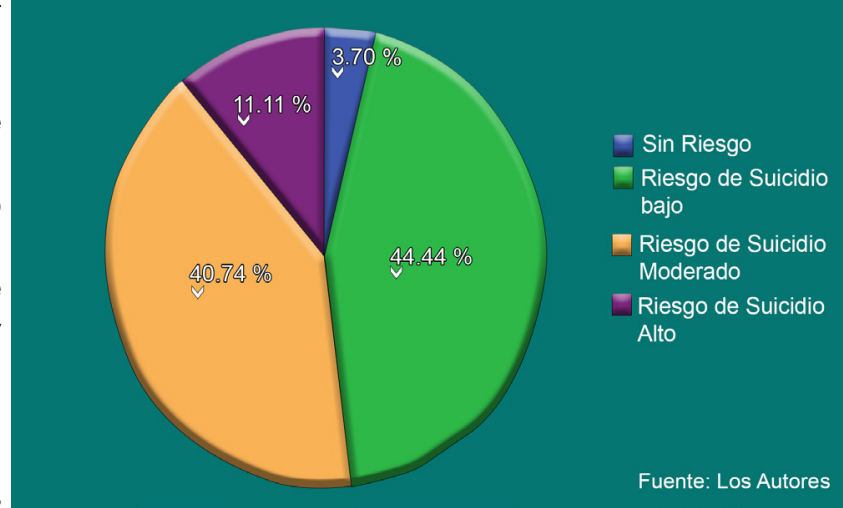
necesario que la persona tenga ayuda de un profesional en salud mental. Por último, un $11.1 \%$ de los participantes se encuentran en nivel de riesgo alto, lo que denota una ideación suicida frecuente, intensa y persistente, asimismo, existen planes específicos de suicidio, e indicadores de intención suicida, existe un método accesible o disponible, lo que evidencia un posible deterioro de autocontrol. 
Riesgo suicida y consumo de canabis: La tabla 1 refleja la relación entre consumo de cannabisy riesgo suicida. Se observa que los participantes con riesgo suicida bajo el $54.2 \%$ tiene un nivel de riesgo moderado por consumo de dicha sustancia.

Tabla 1. Consumo de cannabis y riesgo suicida

\begin{tabular}{|c|c|c|c|c|c|c|c|}
\hline \multicolumn{8}{|c|}{ Cannabis } \\
\hline & & & No consumo & Bajo & Moderado & Alto & Total \\
\hline \multirow{8}{*}{$\begin{array}{l}\text { Riesgo } \\
\text { Suicida }\end{array}$} & \multirow[t]{2}{*}{ Sin Riesgo } & Rencuentro & 2 & 0 & 0 & 0 & 2 \\
\hline & & $\begin{array}{l}\% \text { dentro de } \\
\text { riesgo suicida }\end{array}$ & $100,0 \%$ & $0,0 \%$ & $0,0 \%$ & $0,0 \%$ & $100,0 \%$ \\
\hline & \multirow{2}{*}{$\begin{array}{l}\text { Riesgo de } \\
\text { suicidio } \\
\text { bajo }\end{array}$} & Rencuentro & 7 & 1 & 13 & 3 & 24 \\
\hline & & $\begin{array}{l}\% \text { dentro de } \\
\text { riesgo suicida }\end{array}$ & $29,2 \%$ & $4,2 \%$ & $54,2 \%$ & $12,5 \%$ & $100,0 \%$ \\
\hline & \multirow{2}{*}{$\begin{array}{l}\text { Riesgo de } \\
\text { suicidio } \\
\text { moderado }\end{array}$} & Rencuentro & 8 & 0 & 0 & 3 & 6 \\
\hline & & $\begin{array}{l}\% \text { dentro de } \\
\text { riesgo suicida }\end{array}$ & $36,4 \%$ & $0,0 \%$ & $27,3 \%$ & $36,4 \%$ & $100,0 \%$ \\
\hline & \multirow{2}{*}{$\begin{array}{l}\text { Riesgo de } \\
\text { suicidio } \\
\text { alto }\end{array}$} & Rencuentro & 3 & 0 & 0 & 3 & 6 \\
\hline & & $\begin{array}{l}\% \text { dentro de } \\
\text { riesgo suicida }\end{array}$ & $50,0 \%$ & $0,0 \%$ & $0,0 \%$ & $50,0 \%$ & $100,0 \%$ \\
\hline
\end{tabular}

En cuanto al nivel alto de riesgo suicida, se destaca que el $50.0 \%$ no consumen esta sustancia y la otra mitad de los participantes se encuentran en un nivel alto por consumo de cannabis.

En la tabla 2 se presenta la relación que existe entre consumo de cannabis y riesgo suicida, donde se puede observar que el valor de Prueba exacta de Fisher $\mathrm{P}=15.013, \infty=0.047, \mathrm{X} 2=14.184, \mathrm{gl}=$ $15, \infty=0.212$, según la prueba de Fisher, existe relación entre el consumo de esta sustancia con bajo riesgo suicida.

Tabla 2. Cannabis y Riesgo suicida. Prueba de chi-cuadrado y prueba exacta de Fisher

\begin{tabular}{|l|c|ccccc|}
\hline \multicolumn{2}{|c|}{ Valor } & gl & $\begin{array}{l}\text { Sig. asintótica } \\
\text { (2 caras) }\end{array}$ & $\begin{array}{l}\text { Sig. asintótica } \\
\text { (2 caras) }\end{array}$ & $\begin{array}{l}\text { Significación } \\
\text { exacta (1 cara) }\end{array}$ & $\begin{array}{l}\text { Probabilidad } \\
\text { en el punto }\end{array}$ \\
\hline $\begin{array}{l}\text { Chi-cuadrado } \\
\text { de Pearson }\end{array}$ & $14,184 a$ & 9 &, 116 &, 114 & \\
\cline { 2 - 7 } \\
$\begin{array}{l}\text { Prueba exacta } \\
\text { de Fisher }\end{array}$ & 15,013 & & &, 047 & \\
\hline
\end{tabular}

Fuente: Los Autores

Riesgo suicida y consumo de sedantes: En la tabla 3 se reflejan los resultados de la relación del consumo de sedantes con el riesgo suicida. Para el nivel de riesgo suicida bajo se muestra que la mayoría de los participantes (83.3\%) no son consumidores. 
En el nivel moderado de riesgo suicida, el $68.2 \%$ no consumen sedantes, mientras que un $22.7 \%$ se encuentran en un nivel moderado y un $9.1 \%$ en el nivel alto por consumo de la sustancia antes mencionada. El $33.3 \%$ de los participantes con riesgo suicida alto presentan un nivel de consumo bajo y otro porcentaje similar se ubican en el nivel moderado.

Tabla 3. Consumo de sedantes y riesgo suicida

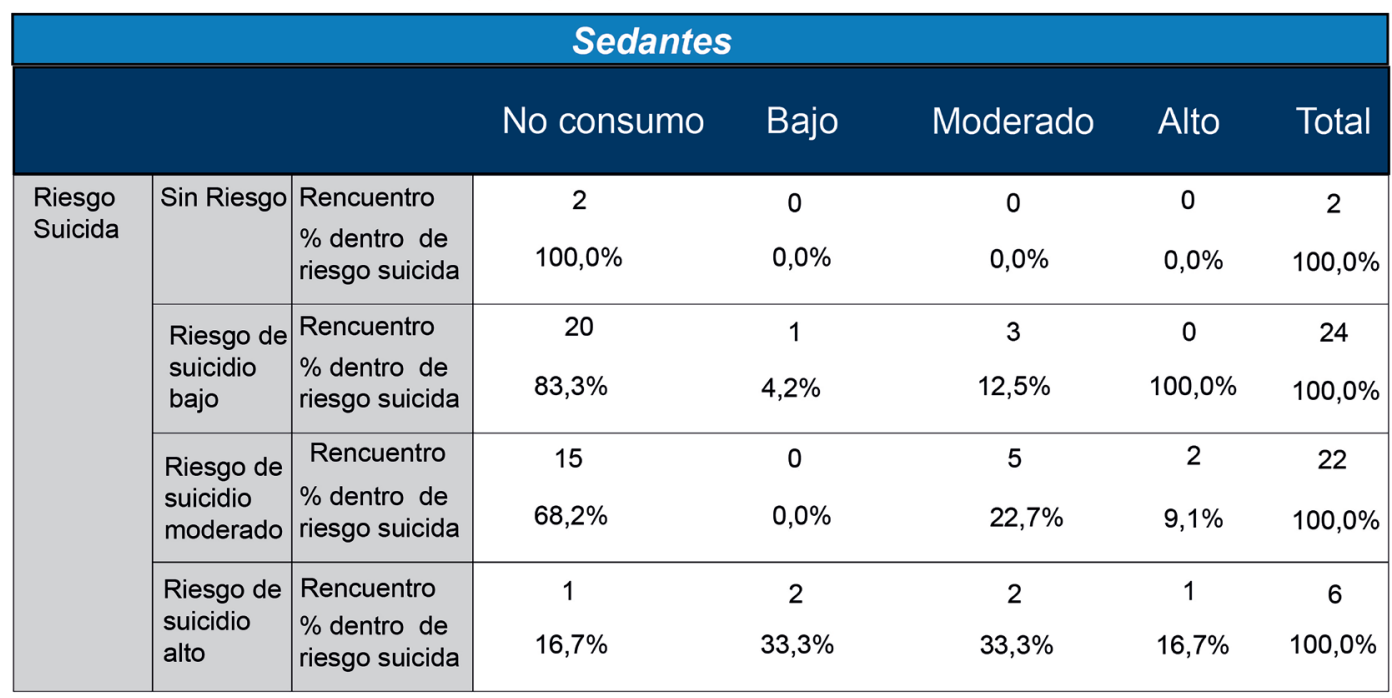

Fuente : Los Autores

La tabla 4 se presenta la relación entre consumo de sedantes y riesgo suicida, donde se puede observar que $\mathrm{X} 2=18.081, \mathrm{gl}=9, \infty=.034$; también, se toman en cuenta los valores de la prueba exacta de Fisher, en donde $\mathrm{P}=16.064, \infty=0.023$, por tanto, se comprueba la relación de consumo de sedantes y riesgo suicida.

Tabla 4. Sedantes y Riesgo suicida. Prueba de chi-cuadrado y prueba exacta de Fisher

\begin{tabular}{|c|c|c|c|c|c|c|}
\hline & Valor & gl & $\begin{array}{l}\text { Significación } \\
\text { asintótica (bilateral) }\end{array}$ & $\begin{array}{l}\text { Significación } \\
\text { exacta (bilateral) }\end{array}$ & $\begin{array}{l}\text { Significación } \\
\text { exacta (unilateral) }\end{array}$ & $\begin{array}{l}\text { Probabilidad } \\
\text { en el punto }\end{array}$ \\
\hline $\begin{array}{l}\text { Chi-cuadrado } \\
\text { de Pearson }\end{array}$ & $18,081 a$ & 9 & ,034 &, 042 & & \\
\hline $\begin{array}{l}\text { Prueba exacta } \\
\text { de Fisher }\end{array}$ & 16,064 & & & ,023 & & \\
\hline
\end{tabular}

\section{Discusión}

Fuente: Los Autores

En relación a los niveles de riesgo que representan las sustancias psicoactivas en la muestra del estudio, las que presentaron mayor consumo y riesgo son las bebidas alcohólicas, tabaco, cannabis, cocaína y sedantes. Las bebidas alcohólicas son las sustancias que más daño genera $n$ en los participantes del estudio, esta representa el mayor riesgo debido a la dependencia que desarrolla la persona. Al ser sustancias legales, su producción, distribución, comercialización, publicidad, venta y consumo están permitidos por la ley (Elizondo, 2005), por lo tanto, en comparación al resto de sustancias, esta se puede adquirir y consumir con mayor facilidad, por lo que la mayoría de usuarios (59.6\%) tienen un nivel de riesgo alto con respecto a esta sustancia. 
En lo referido al consumo del tabaco, la mayoría de los participantes se encuentran en un nivel de riesgo moderado. Según Volkow (2010), la nicotina, que es un compuesto de dicha sustancia, es el mayor responsable de crear la adicción en la persona, esto genera dificultades para dejar de consumir y riesgo para la salud considerables.

En cuanto al consumo de cannabis, el $60 \%$ de los usuarios se encuentran entre un riesgo moderado y alto de consumo, por lo tanto, presenta graves afectaciones en la salud. La predisposición para consumir esta sustancia (Volkow, 2012), va en dependencia de diversos factores, uno de mayor influencia es la historia familiar, la edad de inicio de consumo, el uso de otras drogas y las relaciones con amigos consumidores. Estos factores incrementan las probabilidades del consumo de la sustancia, y una vez que el uso sea repetido se origina la adicción, esto representa para el usuario una dificultad para controlar el consumo aunque este lo desee y tenga consciencia de los efectos negativos que produce.

Otra de las sustancias de mayor consumo y niveles considerados de riesgo por su uso es la cocaína (incluido el crack), de acuerdo con los resultados el $38.8 \%$ de los usuarios se encuentran en un nivel de riesgo alto. Caballero (2005) refiere que tras un consumo inicial de cocaína, muchas personas se detienen por diferentes factores, no obstante, otras personas pasan a un consumo regular y comprenden que a mayor consumo la sensación de euforia será prolongada, esto genera que la persona continúe consumiendo hasta llegar al consumo compulsivo a pesar de las consecuencias adversas.

Referente al consumo de sedantes, el $24.08 \%$ de los participantes del estudio, se encuentran entre un nivel de riesgo alto. Estas sustancias están siendo usadas cada día más para fines no médicos. Uno de los aspectos más relevantes de esta sustancia es la percepción errónea de ser medicamentos prescritos, no son peligrosos aun cuando se usan de forma ilícita.

En los niveles de riesgo alto para el consumo de las diferentes sustancias se puede observar como componentes en común: la tolerancia, dependencia y la activación de los circuitos de recompensa cerebral; cuando éstas sustancias generan el efecto deseado por el consumidor incrementará el deseo de consumirlas (craving) en el período de abstinencia. Desde el enfoque cognitivo-conductual, el desarrollo y mantenimiento del abuso de una o varias sustancias psicoactivas se asocia a los efectos reforzadores (positivos o negativos) que producen sobre los consumidores, lo cual también está relacionado con los procesos cognitivos como las expectativas o creencias sobre los efectos de estas y la falta de habilidades de afrontamiento ante el estrés de los consumidores (Becoña, 2002; Belloch, 2008).

Con relación al nivel de riesgo suicida en los participantes de esta investigación, se obtuvo que el $96.2 \%$ se encuentran en riesgo suicida, esto quiere decir que la persona experimenta ideas suicidas o intentos suicidas (Rocamora, 2013). La mayoría de los participantes se encuentran en un nivel de riesgo suicida bajo, es decir, que únicamente experimentan ideas con una frecuencia baja, esto podría estar relacionado a que la ideación suicida es la forma más común de la conducta suicida y posterior a estas surgen los intentos suicidas (Mingote et al. 2004; Vargas et al., 2015).

Los resultados también reflejan un porcentaje considerable de participantes en riesgo suicida moderado, esto indica que la persona tiene ideas suicidas con una frecuencia alta, hay mayor planificación de cómo llevar a cabo el suicidio, es decir, que los pensamientos tienden a convertirse en acciones. La presencia de riesgo suicida en los participantes se vincula con la desesperanza que pueden estar experimentando, la cual consiste en percibir el futuro de manera 
negativa y de tener la convicción de que nada se puede hacer para mejorar la situación de vida, también existe un estado cognitivo de ambivalencia en donde la persona está motivada por acabar con su vida, pero al mismo tiempo, normalmente tiene deseo de continuar viviendo (Sue et al., 2010), esto explicaría que la mayoría de los participantes se encuentra, en un nivel de riesgo suicida bajo, teniendo únicamente ideas suicidas sin ninguna acción concreta.

Esta desesperanza experimentada por los participantes es explicada desde la teoría cognitiva de la depresión, propuesta por Beck (como se citó en Rocamora, 2013), en esta se afirma que la desesperanza tiene que ver con una triada cognitiva negativa que consiste en tener una visión pesimista de sí mismo, del mundo y del futuro. El individuo tiende a crear categorías mentales en función de sus experiencias vividas, las cuales estructuran y orientan su propia conducta, por lo cual, si esta conducta es desadaptada (el consumo excesivo de una sustancia) la persona va a percibir una imagen distorsionada de su realidad, formará conceptualizaciones negativos sobre sí mismo, su entorno y el futuro; lo que origina desesperanza, depresión y progresivamente la aparición de conductas suicidas iniciando por la ideación.

Uno de los principales hallazgos es la correlación que existe entre dos sustancias depresoras, el cannabis y sedantes con el riesgo suicida. Estos resultados se pueden explicar según los efectos neurofisiológicos (depresores) que generan estas sustancias psicoactivas en el sistema nervioso central, específicamente en la alteración de neurotransmisores que dan origen a una depresión general (Sue et al., 2010), en referencia a esto, Morfin et al. (2015), aseguran que estos efectos representan un factor influyente en la aparición de ideas e intentos suicidas o bien del suicidio consumado.

\section{Conclusiones}

Las bebidas alcohólicas tienen mayor prevalencia en la muestra seleccionada, probablemente esto se deba por la disponibilidad de la sustancia en el medio social.

En cuanto a los niveles de riesgo de consumo, el alcohol presentó un nivel de riesgo alto en la mayoría de participantes, esto indica posibles experimentaciones de problemas graves de salud en los participantes. En relación al uso del tabaco, se reveló un nivel de riesgo moderado en la mayoría de participantes. Además, con respecto al uso de cocaína, se encontró que un mayor segmento de los participantes se encuentra en un nivel de riesgo alto, indicando que este actual patrón de consumo representa un riesgo para su salud.

En el abordaje de los niveles de riesgo suicida, se encontró que la mayoría de los participantes de este estudio, presentan un nivel de riesgo suicida bajo, las personas son capaces de experimentar ideaciones suicidas con frecuencia, intensidad y duración bajas.

Finalmente, el cannabis y los sedantes presentaron correlación con respecto al riesgo suicida, al tener un efecto depresor en sistema nervioso central, pueden tener relación con el surgimiento de ideas como intentos suicidas o bien un suicidio consumado. 


\section{Referencias bibliográficas}

- Aristizábal, C., Cañón, s., Castaño, J., Castaño, L., García, K., Marín, J., Osorno, J., Sánchez, G. (2012). Riesgo suicida y factores asociados en instituciones de rehabilitación para adictos a las drogas en la ciudad de Manizales (Colombia). Red de Revistas Científicas de América Latina y el Caribe, España y Portugal. Recuperado de http://www.redalyc.org/ pdf/2738/273828094002.pdf

- Asociación Americana de Psiquiatría, Manual diagnóstico y estadístico de los trastornos mentales (DSM-5®), 5a Ed. Arlington, VA, Asociación Americana de Psiquiatría, 2014.

- Becoña, E. (2002). Bases científicas de la prevención de las drogodependencias. Madrid, España: Delegación del Gobierno para el Plan Nacional sobre Drogas Secretaría General Técnica.

- Belloch, A., Sandín, B., y Ramos, F. (2008). Manual de psicopatología. Madrid, España: McGraw Hill.

- Caballero, L. (2005). Adicción A Cocaína: Neurobiología, Clínica, Diagnóstico Y Tratamiento. Recuperado de: http://www.fundacioncsz.org/ArchivosPublicaciones/144.pdf

- Casas, M., Bruguera, E., Duro, P., y Pinet, C. (2011). Manual de trastornos adictivos. Madrid, España: Enfoque.

- Elizondo, J. (2005). El Abc Del Alcoholismo. Recuperado de: http://www.revistaadicciones. com.mx/articulos/AA6.htm

- González, C., Juárez, C., Jiménez, A., Montejo, L., Oseguera, G., y Wagner, F. (2015). Ideación suicida y su asociación con drogas, depresión e impulsividad en una muestra representativa de estudiantes de secundaria del estado de Campeche, México. Acta universitaria. Recuperado de http://www.actauniversitaria.ugto.mx/index.php/acta/article/ view/862

- Martínez, J. (2006). Factores asociados a los intentos de suicidio en pacientes atendidos en hospital Roberto Calderón. Managua. Enero 2004 - diciembre 2005 (tesis de maestría). Universidad Nacional Autónoma de Nicaragua, Nicaragua.

- Mingote, J., Jiménez, M., Osorio, R., y Palomo, T. (2004). Suicidio: Asistencia clínica. Madrid, España: Ediciones Díaz de Santos, S.A.

- Morfin, T., \& Ibarra, A. (2015). Fenómeno suicida: Un acercamiento transdisciplinar. Distrito federal, México: El Manual Moderno. 
- Organización Mundial de la Salud (OMS). (2008). Principios delTratamiento de la Toxicomanía . Viena: Autor.

- Organización Mundial de la Salud (OMS). (2011). La prueba de detección de consumo de alcohol, tabaco y sustancias (ASSIST): Manual para uso en la atención primaria. Recuperado de: http://www.who.int/substance_abuse/activities/assist_screening_spanish.pdf.

- Rocamora, A. (2013). Intervención en crisis en las conductas suicidas. Bilabao, España: Desclée de Brouwer.

- Sue, D., Wing, D., y Sue, S. (2010). Psicopatología Comprendiendo la conducta anormal. Distrito Federal, México: CENGAGE Learning.

- Vargas, G., Vasquez, C., Soto, G., y Ramirez, L. (2015). Atención con calidad a las personas que presentan conducta suicida: Manual para profesionales de la salud. Medellín, Colombia: Editorial Alonso Escobar.

- Volkow, N. (2010). Adicción al tabaco. Recuperado de: https://d14rmgtrwzf5a.cloudfront. net/sites/default/files/rrtabaco_0.pdf

- Volkow, N. (2012). La marihuana: Información para los adolescentes. Recuperado de: https:// www.drugabuse.gov/sites/default/files/nida_mj_teenssp.pdf. 


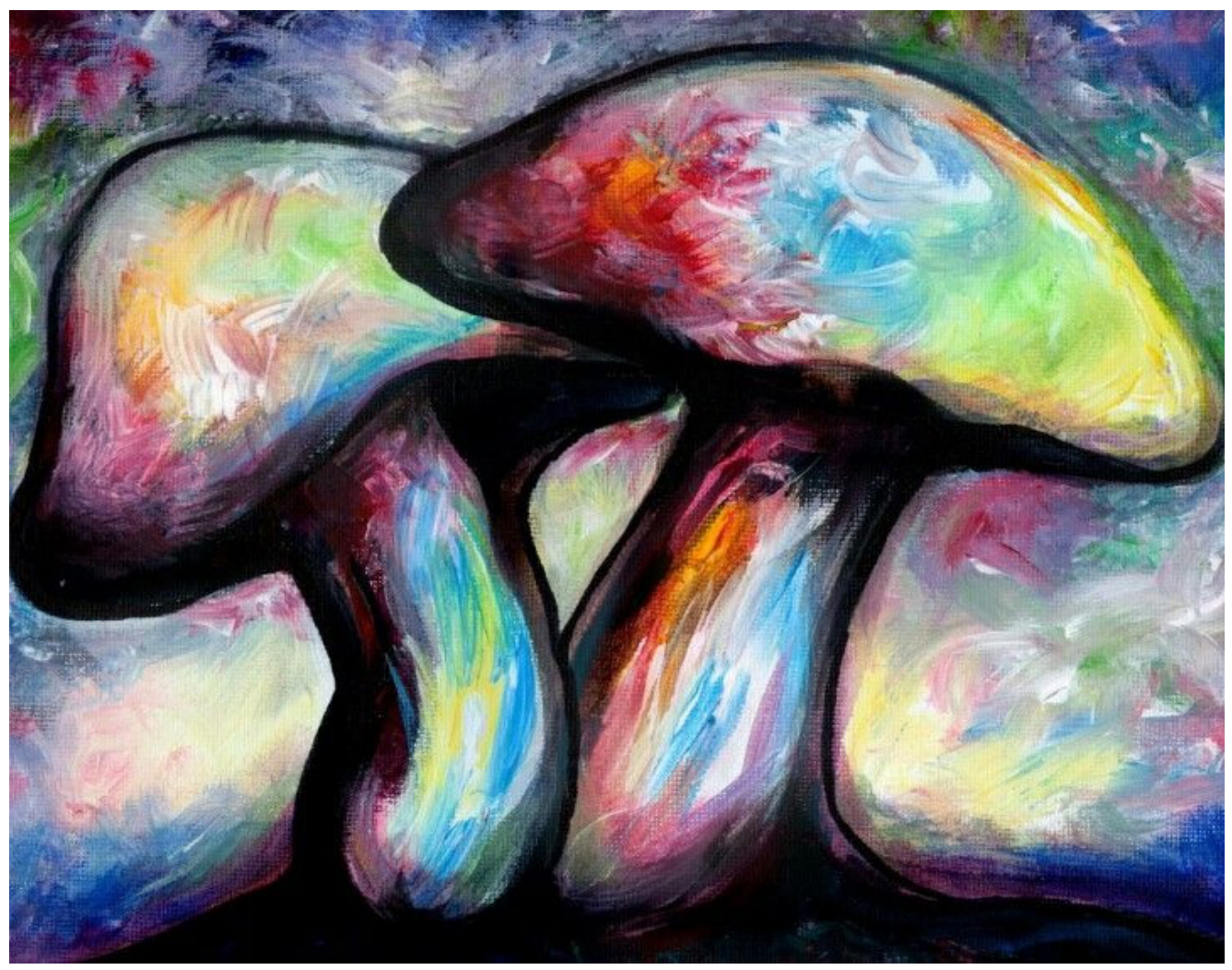

MSc. Dustin Ezequiel Amador Jiménez Master en Psicología Clínica y de la Salud, Licenciado en Psicología, profesor del Departamento de Psicología, Universidad Nacional Autónoma de Nicaragua (UNANManagua).

ORCID ID: 0000-0001-6894-4997

dustin_amador@live.com

Lic. Xochilt María Salinas Pérez.

Licenciada en Psicología, egresada del Departamento de Psicología de la UNANManagua.

ORCID ID: 0000-0003-1847-1217

xoo150508@gmail.com

Lic. Cristhian Josué Pérez López.

Licenciado en Psicología, egresado del Departamento de Psicología de la UNAN- 